\title{
Discussion on Traditional Chinese Medicine Understanding of Renal Cyst
}

\author{
Ningning Li ${ }^{1}$, Ping Fan ${ }^{2 *}$ \\ ${ }^{1}$ The First School of Clinical Medicine, Shaanxi University of Chinese Medicine, Xianyang 712046, Shaanxi Province, China \\ 2Department of Nephrology, Shaanxi Provincial Hospital of Chinese Medicine, Xi'an 710003, Shaanxi Province, China \\ *Corresponding author: Ping Fan, 2448208367@qq.com

\begin{abstract}
The article, from perspective of traditional Chinese medicine (TCM), summarizes etiology as well as pathogenesis, dialectical classification, and treatment method of renal cyst, and concludes that when treating the disease, the first thing to do is to supplement the kidney, then give treatment to the pathogen and tonify qi while attack the pathogen. Additionally, the key of the treatment is to have dynamic observation and timely treatment. In a word, TCM integrated treatment has unique advantage of treating the disease.
\end{abstract}

Keywords: Renal cyst; Deficiency; Stasis

Publication date: September 2021; Online publication: September 30, 2021

\section{Introduction}

Renal cyst is one or more cystic mass with uneven sizes in the kidney, which is not connected with the outside world, and the internal material is mainly liquid or semi-solid liquid. Its nature is benign. The clinical manifestations are low back pain, high blood pressure, low back lump, infection, hematuresis, internal capsule hemorrhage, hydronephrosis, and cyst burst when severe [1]. There is no name of "renal cyst" in traditional Chinese medicine, and it is classified into "lumbago" and "accumulation" according to its characteristics and clinical manifestations. The etiology, pathogenesis and treatment of renal cyst in TCM are summarized as follows.

\section{Explore the etiology and pathogenesis}

"Qisong Yanji, Low Back Pain" states that low back pain has difference between deficiency and reality, and deficiency includes deficiency of kidney essence, Qi and blood. The reality is the invasion of wind, cold and damp heat or trauma caused by phlegm and blood stasis block the blood vessels of the two lumbar meridians, and pain is caused by incompetence. The theory in "Jing Yue's Complete Work" of low back pain is mostly deficiency. Kidney essence and blood Qi gradually deficient, which is unable to normal nourish main and collateral channels, not thrive is painful. "Magic Pivot, The Beginnings of the Hundred Diseases" records that "Suddenly affected by the evil of external cold, sadness and anger, emotional failure, leading to upward inversion of Qi, viscera meridian Qi and blood is not smooth, Qi stagnation, blood coagulation, fluid transport loss, long time not go, accumulation becomes complete." Xili Wu et al. ${ }^{[2]}$ believed that the primary and acquired factors caused Yang Qi damage in the body, and the spleen and kidney were injured. The water transport was not good, and the deficiency of kidney Yang caused the loss of bladder transpiration and vaporization, and the water condensed into sputum. Qi gets warm to go, Yang deficiency makes Qi and blood run not smooth, phlegm and blood stasis intersection obstruction in the vein, blood stasis and phlegm turbidity in the kidney, condensation is not scattered, and finally form cysts. 


\section{Dialectical classification of TCM}

Some scholars ${ }^{[3]}$ divided its TCM syndromes into: Qi stagnation and temperature resistance type, Qi stagnation and blood stasis type, blood stasis and obstruction of collaterals type, accumulated blood stasis type, insufficiency of both the spleen and the kidney type. $\mathrm{Li}$ Luo and Zhibin $\mathrm{Li}^{[4-5]}$ found that deficiency Qi and Yin type as well as dampness and hotness obstruction type were the most common syndromes in patients with renal cysts, while insufficiency of both the spleen and the kidney type as well as dampness, turbidity and stasis type were more common in patients with bilateral and multiple renal cysts.

\section{Traditional Chinese medicine treatment}

Renal cysts should be treated in stages. Yueqing Lu and Boxiang Tang ${ }^{[6-7]}$ both believed that renal cysts in the early stage were mainly caused by solid pathogenic factors such as phlegm turbidity, dampness obstruction and blood stasis. In the medium term, the real evil last for a long time, transform to heat injuring Yin and appeared deficiency and solid inclusion; In the later stage, Qi, blood and Yin and Yang are deficient. On the treatment, early stage it should be given priority to warm phlegm and drink wet, the stage of deficiency and excess should be nourishing Yin and clearing heat, promoting blood circulation and removing stasis, and the later stage should be qi and promoting blood circulation, harmonizing Yin and Yang. The difference is that Mr. Tang practiced qi and blood circulation throughout the course of treatment. Liu Hongfang et al. ${ }^{[8]}$ believed that in the early stage of the disease, the main methods were internal stasis of phlegm drink and collaterals obstruction by blood stasis; and treat with warm phlegm drink, blood circulation and collaterals. In the middle stage of the disease, phlegm and blood stasis can obstruct collateral, and the functions of spleen and kidney are maladjusted. The maladjustment of Qi movement leads to Qi stagnation, which can promote blood flow, and Qi stagnation leads to poor blood operation, astringent blood vessels, increasing blood stasis, and persistent drinking. It is necessary to strengthen spleen and kidney on the basis of eliminating phlegm and dispersing blood stasis. It should be noted that the dosage of this phase should be applied from a small dose, not for a long time, and can be stopped if it is clinically effective. In the later stage of the disease, spleen and kidney are gradually deficient, phlegm and blood stasis block renal collaterals and form accumulatiom. Body evil prosperous while posiitive declines; At this time, we should strengthen the strength of Tonifying the spleen and kidney, and should choose Ginseng Rehmannia Decoction. At this time, the accumulation of cysts is difficult to dissipate. At the same time, drugs for promoting blood circulation and dredging collaterals should be added as appropriate. In the late stage of the disease, both Yin and Yang of the kidney are deficient, damaging the kidney essence, essence does not turn into Qi, kidney Qi turns into division, phlegm turbidity, blood stasis and water stop in the body and cannot be discharged. The treatment should pay attention to tonifying kidney essence and choose SixIngredient Rehmannia Decoction. In this period, we should pay attention to the skillful use of Radix Rehmanniae Preparata, not limited to the use of Herba Taxilli, Radix Achyranthis Bidentatae and other spicy and warm kidney tonifying products.

At present, the main treatment of renal cyst in traditional Chinese medicine is treatment by Chinese herbs. The medication ideas are as follows:

(1) Tonifying the kidney first: the kidney governs water and fluid, the essence, Qi and Yin and Yang of the kidney are sufficient, and the metabolism of body fluid is normal and orderly, especially the sufficient Kidney Yang is the key. Peng An et al. ${ }^{[2]}$ adhered to the principle of "warming and dredging must be the first to control water" and adopted the method of "Warming Yang, activating blood circulation, strengthening spleen and benefiting water" to select Cinnamon Twig and Poria Pill and Chinese Peony Powder to treat renal cyst. Zhigang Tong ${ }^{[9]}$ compared Eliminate Cyst Pill with traditional puncture sclerotherapy and proved that Eliminate Cyst Pill can effectively alleviate the 
discomfort of patients and has a high cure rate. Professor bangben Zheng and Yuling Gou ${ }^{[10-11]}$ both used Six-Ingredient Rehmannia Pill to treat renal cysts, and the clinical feedback was good.

(2) Cure with evil: Professor Pu Gao ${ }^{[12]}$ drew up Wuhai Decoction based on the method of resolving phlegm and removing blood stasis, which is tailored according to the syndrome, and the curative effect is very good. Professor Yulin Wang [13] made good use of the effects of Rhizoma Amorphophalli in removing blood stasis and swelling, reducing phlegm, resolving masses and relieving pain in the treatment of renal cysts. The author found that in the treatment of renal cyst, the products of breaking blood and eliminating syndrome and regulating Qi and eliminating stagnation were mostly added.

(3) Tonification and purgation in combination: Wuyun Wang and Ming Gao ${ }^{[14-15]}$ respectively used tonifying kidney and removing stasis prescription and self-made attack, supplement and eliminating cyst formula to treat patients with renal cyst, with good clinical effect.

\section{Summary}

The clinical manifestations of renal cyst are diverse. The key to treatment is dynamic observation and timely treatment. Traditional Chinese medicine has unique advantages in the comprehensive treatment of renal cyst. At present, there is still a lack of reports on the special traditional Chinese medicine therapies for renal cyst, such as moxibustion and acupoint application, which need further clinical exploration and verification.

\section{Disclosure statement}

The author declares no conflict of interest.

\section{References}

[1] Zhang FR, 2021, Correct Treatment of Renal Cysts is the Key. TCM Healthy Life Nurturing, 7 (5): $17-20$

[2] Wu XL, An P, Shi XM, et al., 2016, Clinical Experience and Typical Medical Cases of Treating Renal Cyst with Warming Yang and Promoting Blood Circulation. Proceedings of 2016 Annual Conference of China Association of Traditional Chinese Medicine Branch of Tonifying Kidney and Activating Blood and the First Summit Forum of Integrated Traditional Chinese and Western Medicine in Diabetic Nephropathy, 91-94.

[3] Zhang LQ, Li PX, Zheng WS, 2000, Complete Book of Nephropathy Diagnosis and Treatment. Beijing: China Medical Science Press, 216-219.

[4] Lou L, 2012, Correlation Analysis of Clinical Features and TCM Syndromes in 300 Patients with Cystic Kidney Disease. Guangdong: Guangzhou University of Chinese Medicine.

[5] Li ZB, 2015, Clinical Analysis and TCM Syndrome Types of 604 Patients with Simple Renal Cyst. Guangdong: Guangzhou University of Chinese Medicine.

[6] Wang QQ, Zhou YL, Lu YQ, 2019, Lu Yueqing's Experience in Treating Renal Cyst. China's Naturopathy, 27(12): 6-7

[7] Tang BX, Wang HB, Ze XY, et al., 2009, Tang Boxiang's Experience in Treating Renal Cyst with Traditional Chinese Medicine. Guide of China Medicine, 7(15): 111-111

[8] Liu HF, Zhang XW, Hu JY, et al., 2018, Experience in Treating Multiple Renal Cysts by Stages in Traditional Chinese Medicine. Global Traditional Chinese Medicine, 11 (3): 395-397

[9] Tong ZG, Wang LX, 2014, Clinical Observation on the Treatment of Renal Cyst with Eliminate Cyst Pill. Guide of Chinese Medicine, (24):283-283. 
[10] Hu JH, Yu ZY. 2019, Zheng Bangben's Experience in the Treatment of Simple Renal Cyst. Journal of Practical Traditional Chinese Medicine, 35 (10): 1272-1273

[11] Gou YL, 2020, Clinical Application of Tonifying kidney and Nourishing Essence in treating Kidney Related Diseases. Chinese Medicine Modern Distance Education of China, 18(18):118-120.

[12] Wen JL, Jin ZH, Wang R, 2017, Professor Gaop's Experience in Treating Renal Cyst with Wuhai Decoction. Information on Traditional Chinese Medicine, 34 (5): 46-47

[13] Wang TT, Xie J, Wang YL, 2020, Wang Yulin's Experience in the Treatment of Simple Renal Cyst. China's Naturopathy, 28 (20): 23-25.

[14] Wang WY. 2007, Observation on the Therapeutic Effect of Bushen Tongyu Xiaoji Recipe in Treating 62 Cases of Renal Cyst. Hebei Journal of Traditional Chinese Medicine, 29 (10): 899

[15] Gao M, Fu MJ, Jiang R, 2007, Clinical Observation on The Treatment of Simple Renal Cyst by Tapping Buxiao Capsule Recipe. Hubei Journal of Traditional Chinese Medicine, 29 (8): 44-44. 